\title{
EMBARAZO Y MALIGNIDAD
}

\author{
Dr. Eduardo Arévalo Burgos* - Dr. Armando Lozano**
}

La circunstancia de que al primero de nosotros le hubiera correspondido dictar una conferencia sobre el tema en el ciclo de las programadas por el Departamento de Obstetricia y Ginecología de la Universidad $\mathrm{Na}$ cional nos indujo a profundizar en él con una revisión de la literatura al respecto que abarca un período de 10 años, desde 1955, y de la estadística del Instituto de Protección Materno-Infantil "Concepción Villaveces de Acosta" desde el año de 1957, cuando encontrándonos de Residentes de la Institución nos correspondió en ese y en los años subsiguientes, atender personalmente algunos casos de $\mathrm{Ca}$. de Cérvix coexistentes con embarazo. Como el Materno-Infantil no es una Clínica de Tumores, la casuística al respecto no es muy abundante en lo que a malignidad en general se refiere en pacientes mujeres que estén 0 nó embarazadas; pero como centro especializado en la atención obstétrica llegan a él muchísimas que son remitidas por hemorragia genital de origen no precisado y la cual no tiene nada que ver en ocasiones con un estado grávidopuerperal, 0 al contrario que por estar embarazadas consultan por pro-

* Profesor Asociado del Departamento de Obstetricia y Ginecología. Univ. Nal.

** Residente 2 del Depto. de Obstetricia y Ginecología de la Univ. Nal. blemas que no tienen ninguna relación con la conducta obstétrica y para cuya solución son remitidas a otros servicios hospitalarios.

Sobre una gran cantidad de enfermedades sistémicas que coinciden con un embarazo o que compliquen una gestación encontramos abundantes referencias en la Literatura: Cardiopatías y Embarazo, Diabetes y Embarazo etc., han sido muy bien estudiadas por diversos autores; mas no sucede lo mismo con las Neoplasias malignas concomitantes con el estado Grávido-puerperal.

Si la literatura mundial no es muy abundante en el tema que nos va a ocupar, lo es mucho menos la nacional de la cual no se han producido sino algunas breves referencias especialmente en relación con el $\mathrm{Ca}$. de Cérvix concomitante con embarazo. Jordan y Darnalt (1) en la 2a. Convención de Obstetricia y Ginecología reunida en Cali en Diciembre de 1955 comentaron 10 casos desde el punto de vista clínico, anatomopatológico y del tratamiento. Gaitán Yanguas (2) en el III Congreso de Obstetricia y Ginecología reunido en lbagué presenta un trabajo con 7 observaciones personales y la revisión de más de 200 casos de Linfomas malignos y su relación con el embarazo. Tirado Vé- 
lez (3) estudia 3 pacientes con $\mathrm{Ca}$. de cuello uterino coincidiendo con una gestación.

En la Convención Internacional contra el Cáncer y Reunión Latinoamericana de Cancerología, reunida en México en Febrero de 1964, de 48 trabajos presentados ninguno de ellos se refirió específicamente al problema de la Malignidad concomitante con el embarazo, es decir a cualquier tipo de Neoplasia maligna de cualquiera de los órganos, que se presente $\mathrm{o}$ se diagnostique en el curso de una Gestación. Solo hay una excepción y son las Neoplasias de la mama en la cual por su evidente relación con el estado grávido-puerperal las referencias son muy abundantes tanto nacionales (7) como foráneas. No ocurre lo mismo con el Coriocarcinoma al decir del cual "El embarazo en cambio, aunque algunos autores, consideren lo contrario, es condición indispensable para que se produzca el coriocarcinoma". De ésta encontramos numerosos trabajos al respecto y seguirán presentándose muchísimos más, no solamente sobre su etiopatogenia sino más que todo sobre su manejo y tratamiento, en abundancia con el brillante porvenir que augura la Quimioterapia.

Tenemos así entonces un solo problema Malignidad y Embarazo que debemos enfocar desde un triple punto de vista. Primero la neoplasia maligna que encontramos en el curso del estado grávido-puerperal en cualquiera de los órganos de la economía, que no tiene una íntima relación con el embarazo en sí pero que complica la gestación misma, o viceversa que la gestación complica la conducta a seguir en el tratamiento de la neoplasia que se ha diagnosticado. Segundo las neoplasias malignas que por tener una localización genital o mamaria, tienen una íntima relación con el estado grávido-puerperal y complican por ello singularmente el embarazo, adquiriendo además normas de conducta excepcionales que es necesario revisar para dejar claramente establecidas. Tercero en fin, el problema de las degeneraciones trofoblásticas que tienen definitivamente un factor etiológico en el estado de gravidez normal o patológico y que tienen su asiento, no como neoplasia propia, sino implantada, casi siempre en alguno de los órganos genitales y cuyos aspectos principales han sido tan exhaustivamente tratados por diversos autores que por la misma razón no serán por nuestra parte objeto de un meticuloso estudio. Nos referiremos pues principalmente a los dos primeros aspectos del problema que encaramos.

\section{MATERIAL Y METODOS}

El material clínico para este trabajo se obtuvo en el Instituto MaternoInfantil que como señalé al principio es una entidad asistencial puramente obstétrica y esta la razón para que el título no fuera Malignidad y Embarazo sino al contrario Embarazo y Malignidad por cuanto prima el hecho de la Gestación a la cual puede sobreagregarse una neoplasia maligna; sin embargo algunos de los casos estudiados procuramos seguirles la pesquisa a través del Instituto $\mathrm{Na}$ cional de Cancerología, donde fueron remitidos, o donde terminaron por recidiva, o en los servicios del Hospital de San Juan de Dios al cual fueron enviadas estas pacientes para tratamiento.

Durante el año de 1957 no se codificó la estadística en el Instituto y fue necesario revisar uno a uno los libros de las salas de partos para dar con aquellos casos de $\mathrm{Ca}$. de cérvix que recibieron tratamiento 
quirúrgico y por ello este año solamente se encuentran dichos casos relacionados, pues aquellos que ingresaron y no fueron intervenidos o que tuvieron el parto vaginal, no figuran, o simplemente estarán anotados como parto espontáneo sin ninguna observación adicional y hubiese sido muy dispendioso por no decir imposible revisar todas las historias clínicas de los ingresos durante ese año. De 1958 en adelante (Marzo) existe codificación y de allí sacamos todos los casos que tuvieron diag- nóstico de neoplasia maligna, para descartar posteriormente aquellas pacientes que no ingresaron en trabajo de parto, como es lo usual, o que no estaban embarazadas, o cuya gestación hubiera terminado por aborto, en cualquier tipo, o fuese este complicado, etc... o no estuvieran en puerperio inmediato. Estos casos pues, de pacientes con algún tumor maligno y con relación evidente con el estado grávido-puerperal forman la casuística del presente estudio.

CUADRO N: 1

\begin{tabular}{llccc}
\hline Años & Ingresos & Partos & Abortos & Embarazos \\
\hline 1957 & no se sacaron datos & & & \\
1958 & 13.598 & 9.281 & 2.134 & 12.112 \\
1959 & 15.871 & 10.648 & 2.251 & 13.829 \\
1960 & 18.254 & 11.916 & 2.750 & 16.170 \\
1961 & 19.987 & 13.165 & 2.979 & 17.626 \\
1962 & 21.339 & 13.911 & 3.520 & 18.981 \\
1963 & 24.198 & 16.247 & 3.852 & 21.760 \\
1964 & 25.283 & 17.751 & 4.170 & 23.350 \\
1965 & 12.614 & 8.917 & 2.238 & 11.651 \\
\hline TOTALES: & 151.144 & 101.836 & 23.984 & 135.479 \\
\hline
\end{tabular}

Cuadro № 1. Por años y total de ingresos, partos, abortos, y embarazos.

\section{CUADRO N: 2}

\begin{tabular}{lccc}
\hline Años & Embarazos & Casos & $\%$ \\
\hline 1957 & - & 3 & $-\overline{0}$ \\
1958 & 12.112 & 5 & 0,041 \\
1959 & 13.829 & 10 & 0,138 \\
1960 & 16.170 & 9 & 0,055 \\
1961 & 17.626 & 6 & 0,034 \\
1962 & 18.981 & 6 & 0,031 \\
1963 & 21.760 & 8 & 0,036 \\
1964 & 23.350 & 7 & 0,025 \\
1965 & 11.651 & 7 & 0,060 \\
TOTALES & 135.479 & $\overline{60}$ & $\overline{0,044}$ \\
\hline
\end{tabular}

Cuadro № 2. Por años y totales de embarazos, casos con malignidad, porcentajes parciales $y$ totales.
Para facilitar el desarrollo haremos una discriminación de la malignidad como sigue:

A) Tumores Malignos en general. 1? Gastro-intestinales y Biliares. 2: Urológicos. 3: Linfomas. 4\% Otros carcinomas. 5: Sarcomas no genitales.

B) Tumores malignos del seno y la esfera genital. 1\% Tumores de seno. $2{ }^{\circ}$ Ca. de vulva y vagina. $3^{\circ} \mathrm{Ca}$. de cérvix y útero. 4\% Ca. de ovarios. 5? Sarcomas genitales.

C) Corioepitelioma.

\section{A) - TUMORES MALIGNOS EN GENERAL}

Bajo este capítulo estudiaremos aquellas neoplasias malignas que se 
diagnostican durante el embarazo y que no tienen una localización específicamente genital o en las glándulas mamarias, pero que complican la conducta a seguir no solamente del curso de la gestación sino de la técnica apropiada de su tratamiento. Las cstadísticas globales de coexistencia de malignidad y embarazo son muy escasas; Pelner, citado por Harbison (4) dá una incidencia aproximada de 0,05\% y agrega que este hecho se explica puesto que el embarazo se encuentra en edades por debajo de los cincuenta años mientras que el más alto índice de malignidad se encuentra en general por cncima de esta edad lo cual hace que la coexistencia de estas dos condiciones sea bastante rara. $H$. Close Hessoltine y Myrna F. Loth de la Univ. de Chicago (5) coleccionaron 33 casos de malignidad en los cuales el diagnóstico fue hecho antes o confirmado durante el embarazo; de éstos, 8 tuvieron una enfermedad sarcomatosa, hubo 25 carcinomas de los cuales 12 fueron del cérvix, 6 del seno, 3 del recto, 1 del colon, 2 en la cara y 1 del tiroides. Betson J. R. y Golden M. L. en Charity Hosp. de Luisiana en New Orleans (6) en Abril de 1961 recolectaron 85 casos de cáncer y embarazo en una revisión que abarca desde 1939 en esa Institución, excluyeron de este estudio los melanomas malignos y todas las formas de leucemia, de su trabajo tomamos los siguientes cuadros que muestran los tipos de malignidad cncontrados, las localizaciones, promedios do edad y oportunidad del diagnóstico en relación con el embarazo: Cuadros Nos. 3, 4, 5 y 6.

En nuestra serie encontramos 60 casos del I. M. I. discriminados así: Cuadro № 7. Para ilustración mayor agregamos un caso de la Clínica San Pedro Claver de un Liposarcoma del muslo.

\section{CUADRO NN 3 \\ BETSON Y GOLDEN}

\begin{tabular}{|c|c|c|c|}
\hline \multirow{2}{*}{\multicolumn{2}{|c|}{$\begin{array}{l}\text { CARCINOMA } \mathbf{7 4} \text { casos } \\
\text { Cérvix (total } 30 \text { casos) }\end{array}$}} & \multicolumn{2}{|c|}{$\begin{array}{l}\text { Promedio de edad } \\
30-36 \text { años }\end{array}$} \\
\hline & & & \\
\hline \multirow{2}{*}{\multicolumn{2}{|c|}{$\begin{array}{l}\text { no invasivo } \\
\text { invasivo }\end{array}$}} & 19 & casos \\
\hline & & 11 & " \\
\hline \multicolumn{2}{|c|}{ Mama } & 16 & " \\
\hline \multicolumn{2}{|l|}{ Tiroides } & 7 & " \\
\hline \multicolumn{2}{|l|}{ Recto } & 5 & $"$ \\
\hline \multicolumn{2}{|l|}{ Riñón } & 4 & $"$ \\
\hline \multicolumn{2}{|l|}{ Ovario } & 4 & " \\
\hline \multicolumn{2}{|l|}{ Vulva } & 3 & "' \\
\hline \multicolumn{2}{|l|}{ Colédoco } & 1 & " \\
\hline \multicolumn{2}{|c|}{ Apéndice } & 1 & $"$ \\
\hline \multicolumn{2}{|c|}{ Gland. Sub-maxilar } & 1 & " \\
\hline \multicolumn{2}{|c|}{ Gland. lacrimal } & 1 & " \\
\hline \multicolumn{2}{|c|}{ Nariz epidermoide } & 1 & " \\
\hline \multicolumn{2}{|l|}{ TOTAL } & $\overline{74}$ & ", \\
\hline
\end{tabular}

\section{CUADRO № 4}

BETSON Y GOLDEN

\begin{tabular}{|c|c|c|c|c|}
\hline SARCOMAS & 11 & casos & $\begin{array}{r}\text { Promedi } \\
26,3\end{array}$ & $\begin{array}{l}\text { - de edad } \\
3 \text { años }\end{array}$ \\
\hline \multicolumn{3}{|l|}{ Hodgkins } & \multicolumn{2}{|c|}{4 casos } \\
\hline Osteogénicos & & & \multicolumn{2}{|c|}{$2 "$} \\
\hline \multicolumn{3}{|l|}{ Cels. gigantes } & \multicolumn{2}{|l|}{2} \\
\hline \multicolumn{3}{|l|}{ Leiomiosarcoma } & \multicolumn{2}{|l|}{1} \\
\hline \multicolumn{3}{|l|}{ Poliposarcoma } & 1 & $"$ \\
\hline \multicolumn{3}{|l|}{ Linfosarcoma } & \multicolumn{2}{|l|}{1} \\
\hline TOTAL & & & $\overline{11}$ & ", \\
\hline
\end{tabular}

La paciente más joven en nuestra serie tenía 17 años de edad y corresponde al caso $\mathrm{N}$ : 38 una Primigestante con un embarazo de más o menos 38 semanas de evolución que presentaba un $\mathrm{Ca}$. basocelular del labio (oral). Las de mayor edad cran de 45 años, tres pacientes grandes multíparas, dos con aborto retenido y una con aborto incompleto y todas con $\mathrm{Ca}$. de cérvix estados III (dos) y IV (una) el promedio de edad en nuestras pacientes fue de 33-35. El promedio de gestaciones fue de 7,35 en 59 pacientes, en una de ellas dejó de anotarse el dato en la corres- 
CUADRO № 5

BETSON Y GOLDEN

\begin{tabular}{|c|c|c|c|c|c|}
\hline Diagnóstico & Antes & Durante & A término & Puerperio & Total \\
\hline \multicolumn{6}{|l|}{ CARCINOMAS } \\
\hline \multirow{2}{*}{$\begin{array}{c}\text { Cérvix no invasivo } \\
\text { invasivo }\end{array}$} & 2 & 14 & 2 & 1 & 19 \\
\hline & - & 9 & 2 & - & 11 \\
\hline Mama & 5 & 9 & 1 & 1 & 16 \\
\hline Tiroides & 4 & 3 & - & - & 7 \\
\hline Recto & 1 & 4 & $\ldots$ & - & 5 \\
\hline Riñón & 1 & 3 & -- & -- & 4 \\
\hline Ovario & - & 2 & 1 & 1 & 4 \\
\hline Vulva & - & 2 & 1 & - & 3 \\
\hline Colédoco & - & 1 & - & - & 1 \\
\hline Apéndice & - & - & - & 1 & 1 \\
\hline Gland. submaxilar & - & 1 & - & - & 1 \\
\hline Nariz epidermoide & - & 1 & - & - & 1 \\
\hline TOTALES & 13 & 50 & 7 & 4 & 74 \\
\hline
\end{tabular}

CUADRO N 6

BETSON Y GOLDEN

\begin{tabular}{|c|c|c|c|c|c|}
\hline Diagnóstico & Antes & Durante & A término & Puerperio & Toial \\
\hline $\begin{array}{l}\text { Carcinomas } \\
\text { Sarcomas }\end{array}$ & 13 & 50 & 7 & 4 & 74 \\
\hline Hodgkins & 2 & 2 & - & - & 4 \\
\hline Osteogénico & 1 & 1 & - & - & 2 \\
\hline Cels. gigantes & - & 2 & - & 一 & 2 \\
\hline $\begin{array}{l}\text { Leiomiosarcoma } \\
\text { Pólipo cervical }\end{array}$ & - & 1 & - & - & 1 \\
\hline sarcomatoso & - & 1 & - & - & 1 \\
\hline Linfosarcoma & 1 & 一 & - & - & 1 \\
\hline TOTALES & 17 & 57 & 7 & 4 & 85 \\
\hline
\end{tabular}

CUADRO NN 7 AREVALO $Y$ LOZANO

\begin{tabular}{|c|c|c|}
\hline $\begin{array}{l}\text { Discriminación de la } \\
\text { malignidad }\end{array}$ & $\begin{array}{c}\text { Edad } p \\
33-3\end{array}$ & $\begin{array}{l}\text { promedio } \\
35 \text { años }\end{array}$ \\
\hline $\begin{array}{c}\text { Cérvix Ca. escamo-celular } \\
\text { Adenocarcinoma }\end{array}$ & $\begin{array}{r}42 \\
1\end{array}$ & casos \\
\hline Coriocarcinoma & 4 & " \\
\hline Corioadenoma destruens & 2 & "' \\
\hline Carcinoma de seno & 4 & " \\
\hline Adenocarcinoma de recto & 1 & " \\
\hline Rabdomiosarcoma de cérvix & 1 & " \\
\hline $\begin{array}{l}\text { Ca. basocelular de labio } \\
\text { Ca. papilar retroperitoneal metas- } \\
\text { tásico de posible origen }\end{array}$ & 1 & " \\
\hline vesical & 1 & $"$ \\
\hline Ca. anaplásico de nariz & 1 & $"$ \\
\hline $\begin{array}{l}\text { Ca. escamocelular del muslo } \\
\text { Ca. de gland. parótida con }\end{array}$ & 1 & " \\
\hline metástasis cerebrales & 1 & " \\
\hline TOTAL & 60 & " \\
\hline
\end{tabular}

pondiente historia; el promedio de paridad fue de 5,77 en el mismo número de enfermas. Quince pacientes ingresaron y se les hizo el Diagnóstico de Malignidad con embarazos de más de 38 semanas de evolución, el promedio de semanas de gestación para 52 pacientes fue de 25,57, tres eran puérperas de 9 días a cuatro meses, una ingresó con un aborto incompleto y sin dato de amenorrea, otra con un aborto retenido, una más con amenorrea de 6 meses después de aborto molar y era un Coriocarcinoma con Quistes Luteínicos y por fin en dos no pudo conocerse ningún dato al respecto. De las 52 pacientes con dato en semanas de la 
CUADRO N 8

AREVALO Y LOZANO

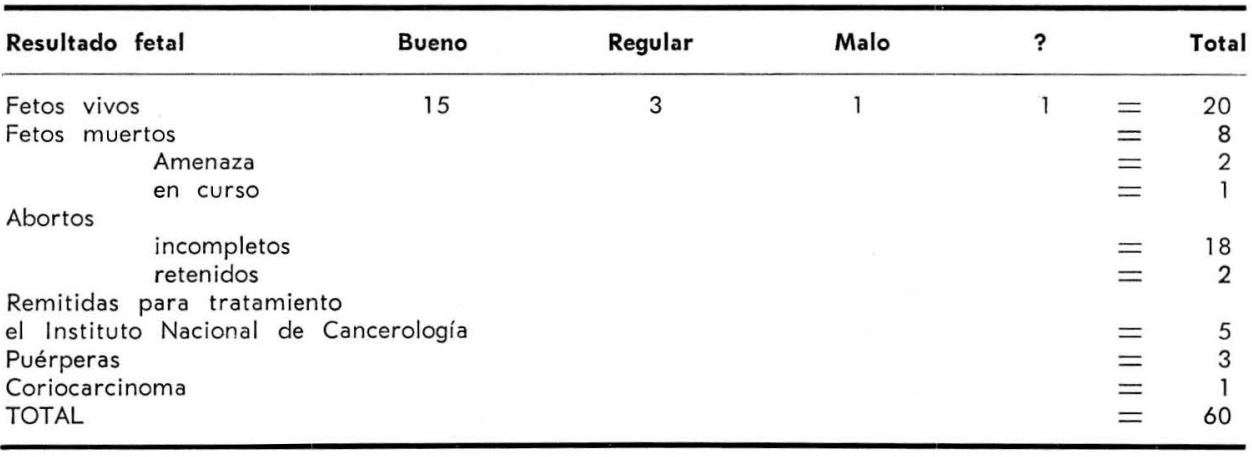

gestación a su ingreso, 20 tenían fetos vivos y de éstos los resultados fetales fueron buenos en 15, regulares en 3 y malo en uno que murió por síndrome de insuficiencia respiratoria. Cuadro No 8; y una paciente con un embarazo de más o menos 32 semanas con feto vivo, diagnóstico de $\mathrm{Ca}$. de seno que rehusó todo tratamiento y abandonó el Instituto. Ocho pacientes ingresaron con feto muerto, veintitres pacientes fueron clasificadas como Aborto, cinco fueron remitidas al Instituto Nal. de Cancerología por considerarse intratables dentro del Materno-Infantil. En relación con la forma de terminación del embarazo hubo 27 partos, 17 Cesáreas Electivas por Malignidad, 9 Partos espontáneos y 1 Histerectomía Total con feto en útero en una Multi VII con embarazo de 40 semanas con feto muerto e infección amniótica severa y $\mathrm{Ca}$. de cérvix. No se practicaron Cesáreas por indicación obstétrica.

Los síntomas, exámenes complementarios, diagnóstico, tratamiento, resultados maternos, complicaciones y controles los describiremos en cada uno de los grupos de malignidad en que hemos dividido el trabajo.
1. Gastrointestinales o biliares. Los carcinomas de Vías Digestivas son muy raras complicaciones del embarazo por la misma razón que anotamos al hablar de los tumores malignos en general, no coinciden las edades picos de unos y otros; sinembargo, encontramos algunos casos de cóIon y recto, 4 en la serie de Heseltine y Loth (5), 5 en la serie de Betson (6) y 1 del Colédoco, y Apéndice en la misma; tan exóticos serán que Jennings, citado por Greenspan y Lesnick (8) en 1952 solo pudo encontrar 77 casos reportados en la literatura mundial desde 1837; igualmente MacBeth, citado por Hárbison (4) agrega un caso más de un Adenocarcinoma de la ampolla de Vater. En nuestra serie el Caso № 1 presentaba un Adenocarcinoma del Recto y lo describiremos en detalle más adelante. Todos los autores están de acuerdo en que no existe ninguna influencia del embarazo en relación con el crecimiento tumoral, este es inherente al neoplasma mismo y desde luego tampoco tiene la gravidez una acción benéfica sobre su evolución. En cambio la sintomatología sí puede ser enmascarada por los disturbios que acompañan a la gravidez tales como dolores abdomi- 
nales difusos, cambios en el hábito intestinal, constipación o crisis diarreicas, etc. Sinembargo, continúa teniendo el mismo valor sospechoso cualquier hemorragia anal que se presente y no existe ninguna contraindicación por el embarazo para practicar un examen proctosigmoidoscópico. En los dos casos citados de Carcinoma de las vías biliares el síntoma común ha sido la Ictericia que hizo sospechar un proceso inflamatorio canalicular inicialmente y luego obstructivo; a pesar del temor que puede originar emplear el radiodiagnóstico en los primeros meses de la gestación no hay justificación para aplazarla en cualquier época en que se crea necesaria. Las líneas generales de tratamiento tienen una variación sobre bases estrictamente individuales en lo que se relaciona con problemas éticos, morales y personales. Si el Cáncer es descubierto en los primeros meses de gestación y es clínicamente curable debe tratarse por los medios usuales, cirugía, fisioterapia con protección adecuada o quimioterapia, corriendo los riesgos de provocar el aborto como con la Roentgenterapia o ciertas substancias quimioterápicas que se han descrito con alguna acción abortiva como la aminoptirina, a pesar de que su uso está actualmente muy restringido en las neoplasias digestivas; 0 posibles deformaciones fetales en embarazos avanzados que van desde la pérdida del cabello en ciertas zonas hasta la microcefalia. En cualquier edad de la gestación si se presentan fenómenos obstructivos debe procederse a la cirugía derivativa. En el último trimestre del embarazo cuenta ya mucho la vida fetal debiéndose dejar éste hasta su máxima viabilidad antes de emprender una terapéutica radical. No existe contraindicación para el parto vaginal a menos que la localización de la neoplasia en recto-sigmoide implique una distocia del canal del Parto que deberá entonces efectuarse por Cesárea o que haya necesidad de derivar en cuyo caso se aprovecha el mismo acto quirúrgico para practicar las dos. Si la resección es posible, se prefiere practicarla semanas después en el puerperio.

El pronóstico no es mejor ni peor que en pacientes no embarazadas y solamente por el retardo que implica muchas veces el diagnóstico oportuno se puede ver ensombrecido.

CASO No 1. H. C. No 41.464 del I. M. I. E. F. Paciente que ingresa el VI-18/57 de 28 años de edad G1. Pl., con una gestación a término consultando por dolor rectal y deposiciones con sangre de más o menos 5 meses de evolución. Feto vivo. Se practicó rectosigmoidoscopia y se hizo diagnóstico de Ca. de recto. Como exámenes complementarios. Se practicaron Cistoscopia y Radiografía de tórax. Parto por Cesárea con buenos resultados fetales, transversostomía en el mismo acto operatorio; se comprobó el Diagnóstico de Adenocarcinoma rectal y la paciente se pasó al Hospital San Juan de Dios para tratamiento radical el 7 de Septiembre de 1957. No tiene controles en el I. M. I.

2: Urológicos. Las neoplasias malignas renales son extremadamente raras en las mujeres jóvenes; su mayor frecuencia en el hombre está en una relación de 3.2 a 5.1 para los dos sexos; por estas razones la coexistencia de malignidad renal con embarazo es una condición de muy rara ocurrencia. Guttmacher y Revinsky citados por Fetter y Koppel (9) en una revisión de 20.000 pacientes obstétricas en 6 años en el Mt. Sinai Hosp. New York, no encontraron ninguna neoplasia renal o vesical.

En la serie de Betson (6) ya citada, reporta 4 casos de $\mathrm{Ca}$. del riñón y Waddington (10) describe un caso 
de Ca. de riñón coexistiendo con embarazo. Los mismos Fetter y Koppel (9) reunieron una serie de 15 casos en una revisión de la Literatura desde 1922 hasta 1963, en ella figuran dos Tumores de Wilms (teratomas), cuatro Hipernefromas (tumores mesenquimatosos de origen glandular) siete carcinomas diversos (adenocels-granulosas y cels-claras) y dos derivados de epitelios atípicos. Los síntomas cardinales son la hematuria macro o microscópica, el dolor a veces de tipo cólico por el paso de un coágulo a través del uréter inferior y la aparición de masa en la región lumbar. La cistoscopia, pielografía retrógrada y demás exámenes urológicos usuales no están contraindicados en el embarazo ni aún el radiodiagnóstico con protección adecuada de la pelvis en cualquier edad de la gestación y el tratamiento debe ser emprendido como si no existiera el embarazo, éste no influye ni favorable ni desfavorablemente sobre el pronóstico. No tenemos ningún caso en nuestra serie.

3 Linfomas. En este grupo de malignidad ocurren cosas muy importantes en relación con el embarazo. En primer lugar aunque en la mujer es menos frecuente que en el hombre la enfermedad de Hodking en proporción de una por cada 2,5 sinembargo su máximo de incidencia se encuentra durante la edad reproductiva y además según Gaitán Yanguas (2) "En nuestro medio es un poco más común, pues, como lo hemos mostrado en nuestro trabajo sobre el tema, los linfomas ocupan el cuarto puesto de frecuencia entre todos los cánceres y a su vez el Hodgkin representa el $34 \%$ de los linfoblastomas". Por otra parte si bien los linfosarcomas malignos por su evolución tan grave y la alteración que producen del estado general hacen que el embarazo sea menos posible, no existe un acuerdo unánime sobre la influencia de éste en la evolución del neoplasma. Algunos, entre ellos el mismo Gaitán Yanguas sostiene que el embarazo es desfavorable para ambas entidades y así lo demuestra en su trabajo. Otros como Barry, Diamond y Gravers (11) sostienen que su influencia nociva se concentra en el puerperio, es decir, que no tiene influencia sobre la evolución del embarazo en sí y otros como Hennessy y Rotino (12) en 35 casos que presentan de Hodgking y embarazo concluyen que éste no tiene ninguna influencia sobre la incidencia, las exacerbaciones ni afecta en forma adversa el tiempo de supervivencia. La frecuencia se ha establecido aproximadamente en uno por cada 6.000 embarazos. El tratamiento depende del grado de evolución de la enfermedad y la edad de la gestación, algunos lo difieren hasta el tercer trimestre, otros lo inician en forma inmediata por escisión quirúrgica de las masas removibles, radioterapia intensa con adecuada protección fetal para evitar las anomalías en el desarrollo; evitando la aplicación de drogas citotóxicas por este mismo motivo. Gaitán Yanguas concluye 1: que debe desaconsejarse el embarazo en mujeres que estén en período de evolución activa del Hodgking. 2: Que debe tratarse intensamente cuando se presenta el embarazo durante períodos activos de éste. 3. Que no es desaconsejable el embarazo en los períodos inactivos de la enfermedad puesto que las posibilidades de recaídas son las mismas que en cualquier otra mujer no embarazada aunque hace la salvedad de que siendo esta enfermedad de evolución muy desfavorable no es conveniente someterla al riesgo de una recaída que son siempre de peor pronóstico. 
4. Otros Carcinomas. Mencionaremos aquí los siete casos de $\mathrm{Ca}$. de Tiroides de la serie de Betson (6) en los cuales ni el embarazo ni la neoplasia tuvieron entre sí relaciones de complicación, todas las pacientes tuvieron parto vaginal normal y todas recibieron tratamientos por $\mathrm{Ra}$ yos $X$ y Tiroidectomía, dos recibieron Quimioterapia adicional y una Yodo Radioactivo como tratamiento paliativo a pesar de que algunos (5) lo contraindican formalmente puesto que estos isótopos cruzan la barrera placentaria produciendo alteración del tejido tiroidiano fetal.

De acuerdo con Pack y Scharnagel, citados por Peck y Kantor (13) los nevus pigmentarios son extraordinariamente sensitivos a las influencias estimulantes de las hormonas pituitaria, adrenal y gonadales y este hecho debe ser muy tenido en cuenta por el obstetra con el fin de prevenir la aparición de un Melanoma en aquellos que están expuestos a trauma por posición anatómica o por ocupación, aquellos que durante el embarazo cambian de tamaño, alteran su uniformidad 0 intensidad de pigmentación, se ulceran, duelen o sangran. Barón (14) aunque dá una incidencia de 1 en 122.000 embarazos, presenta dos casos en los cuales la influencia del embarazo fue notablemente nociva sobre ellos pues ambas pacientes murieron una con metástasis abdominales y la otra con ganglios axilares metastásicos.

Debemos agregar aquí los casos solitarios de nuestra serie a saber: (Cuadro Ni. 7).

EI CASO No 38 de una Primigestante de 17 años de edad con 38 semanas de gestación, feto vivo que ingresa en trabajo, tiene un parto espontáneo normal, masculino de 2.700 gs. de peso $10 / 10$ de Apgar; a dicha paciente se le diagnostica un $\mathrm{Ca}$. basocelular del la- bio oral y se remite en el puerperio al Instituto Nacional de Cancerología para tratamiento.

El CAso No 52 una G. 10 P. 7 A 2 de 37 años de edad con una gestación de 33 semanas aproximadas, feto vivo, que ingresa por dolor lumbar tipo cólico, disuria, polaquiuria y hematuria, se estudió con exámenes de orina, la urografía mostró una exclusión renal derecha, la cistoscopia, compresión extrínseca de vejiga. Se hizo una Cesárea extemporánea $y$ se obtuvo feto vivo, femenino de 2.500 gs. de peso, Apgar 4/10, se tomó biopsia de un tumor retroperitoneal que se reportó como Ca. papilar con metaplasia escamosa posiblemente originario de vejiga; la paciente falleció, no se hizo autopsia, la niña salió en buenas condiciones del Instituto.

El CASO No 57 una G. 8 P. 7 de 28 años de edad con embarazo de 30 semanas aproximadamente que es enviada del Instituto de Cancerología por estar en trabajo de parto que tiene en forma espontánea con feto muerto. La paciente presentaba un Ca. anaplásico de nariz y se remitió nuevamente a Cancerología.

Y por último el CASO $\mathbf{N}^{\circ} \mathbf{5 9}$ una G. 2 P. 2 puérpera de 9 días de 22 años de edad que ingresa por hemorragia genital por retención de restos y con un Ca. de parótida con metástasis cerebrales. Se practicó Revisión Uterina, se dió de alta del Instituto sin que se sepa nada de ella.

Como observamos, en estos casos nada tuvo que ver la Neoplasia estrictamente en la evolución del embarazo pues ni siquiera en la de feto muerto podemos encontrar una relación de causa a efecto. Una Cesárea extemporánea, posiblemente por el mal estado general materno, los otros fueron partos espontáneos. Tampoco podemos deducir que el embarazo haya tenido influencia sobre la evolución tumoral. En lo que se refieren a tumores conjuntivos extragenitales no tenemos casuística pero queremos presentar aquí un caso del Instituto Colombiano de Seguros Sociales: 
Una paciente de 23 años G. 4 P. 1 A. 2 embarazo en evolución de 8 meses que ingresa por presentar tumoración en cara posterior de muslo derecho, en interconsulta con Cirugía, aconsejan tomar biopsia e inducir el parto. La biopsia reportó un Liposarcoma Mixomatoso de bajo grado de malignidad. Se intentó inducción que no prendió, la paciente tuvo luego un parto espontáneo normal y se le dió de baja de la Clínica para resolver su problema neoplásico. ,

\section{B) - TUMORES MALIGNOS DEL SENO Y LA ESFERA GENITAL}

Estas neoplasias que tienen su asiento en los órganos reproductivos constituyen por sí mismas un hecho notable. En primer lugar muchas hacen difícil el embarazo y esterilizan la paciente como sucede por ejemplo en las Neoplasias de Ovario y algunas de genitales externos, tanto por el trastorno funcional que generan como por la alteración misma del estado general que repercute sobre la fertilidad o por su misma presencia física como en los Sarcomas de vagina y cuello o por la infección secundaria siempre presente. Además tampoco es la edad reproductiva femenina la de más alta incidencia de malignidad en forma tal que mientras ésta aumenta la fertilidad decrece, por esto la coexistencia de las dos no es muy frecuente lo cual hace más interesante el tema y lo convierte no propiamente en una complicación del embarazo sino en una complicación con embarazo. En segundo lugar parece un hecho aceptable que el neoplasma "quema etapas" en su evolución con un embarazo cuando tiene su asiento en órganos de la esfera genital o en la glándula mamaria; de otra parte la neoplasia actúa sobre la evolución normal del embarazo y llega hasta imponer conductas obstétricas determinadas en la conducción del parto que van en be- neficio del feto $y$ del tratamiento más adecuado de la neoplasia misma con miras a obtener una mayor supervivencia materna. Si a esto agregamos que la paciente embarazada y en control prenatal es una "paciente cautiva" como la llama McCall (15) en quien podemos utilizar todos los modernos métodos de investigación del cáncer para llegar a un Diagnóstico Precoz, hallaremos entonces la utilidad tan extraordinaria que para el Obstetra tiene el familiarizarse con estos hechos.

19. Tumores malignos del seno. Sobre esta neoplasia encontramos una muy abundante literatura nacional y foránea con numerosas e importantes series de casos revisados desde el punto de vista diagnóstico, tratamiento y supervivencia de 5 años. Vélez y Jubiz (7) de la Fac. de Medicina de la Universidad de Antioquia consideran 13 pacientes con $\mathrm{Ca}$. de seno en 10 años, 1952-1962 del Servicio de Maternidad del Hospital de San Vicente de Paúl sobre 64.471 ingresos. Taylor en 1955 (16) revisa 1.375 casos de $\mathrm{Ca}$. de seno y embarazo de una colección de 144 artículos publicados en la literatura mundial, de sus conclusiones extractamos los siguientes datos:

19. Frecuencia. 3 casos por cada 10.000 embarazos, semejante a la que da Greenspan, Gerson y Lesnick (8) de un caso en cada 3.200 embarazos; mientras que en relación con el Ca. de seno dá una frecuencia de 3 casos con embarazo por cada 100 Carcinomas.

2 . La rata de supervivencia de cinco y diez años ha venido mejorando desde 1941 con un promedio de $21,5 \%$ de los casos sin metástasis el cual se empobrece de 5 a 20 veces en los casos con metástasis. 
3. El promedio de edad fue de 35,8 años.

4․ El retardo entre el diagnóstico y la iniciación del tratamiento en las embarazadas fue en promedio de 3,1 meses.

5․ El pronóstico es más pobre en las pacientes cuyo diagnóstico se hizo en el segundo y tercer trimestre del embarazo que en aquellas tratadas durante el primer trimestre, o durante la lactancia.

Holleb y Farrow del Memorial Hospital For Cancer, New York (17) revisan igualmente la literatura al respecto y presentan una serie de 283 casos; describen las alteraciones funcionales y estructurales de la glándula mamaria durante la gestación y la lactancia, dividiéndola en cuatro períodos desde el momento de la concepción hasta cuando la glándula vuelve a su estado de reposo funcional a saber: proliferativo, secretor, lactación e involución. La forma en que las hormonas que promueven y regulan estas fases en el seno normal puedan cambiar la evolución en el Cáncer mamario no es conocida y su acción no ha podido ser comprobada, no existe evidencia positiva de un aumento de la actividad mitósica - que el carcinoma se haga más anaplásico durante la fase proliferativa, sinembargo, la disminución del estroma fibroso de la glándula y el aumento de la vascularización se ci$\tan$ como elementos que pueden favorecer el crecimiento local del tumor y la extensión metastásica aunque es difícil de confirmarlo clínicamente.

En la serie citada dividieron las pacientes clínicamente en 3 grupos: 1․ Cáncer de seno operable. 2 Cáncer de seno que a su ingreso había sido sometido a mastectomía radical o simple, que no tenían evidencia de recurrencia y que ellos denominan Profilaxis de Cáncer de seno y 3 ? Cáncer de seno inoperable. $68 \%$ eran casos operables, 73 pacientes eran inoperables y 17 del grupo profiláctico. El promedio de edad fue de 33 años; $9 \%$ eran primigestantes, $73 \%$ entre 1 y 3 embarazos, $16 \% 4$ a 6 embarazos y $2 \%$ más de 7 ; 151 casos del seno izquierdo y 129 del derecho, cuatro pacientes en ambos; 38 pacientes recibieron radioterapia antes de la cirugía radical pero la gran mayoría de las tratadas por mastectomía recibieron radioterapia post-operatoria. De las 73 pacientes inoperables la supervivencia entre la admisión y la muerte fue de un mes a 3 años y 7 meses, el promedio fue de 7 meses. No analizan el número de pacientes profilácticas por lo bajo. De las 193 pacientes operables, 28 estaban en el primer trimestre y 24 de ellas fueron sometidas a mastectomía radical, las otras 4 fueron irradiadas o rehusaron tratamiento, la supervivencia de 5 años fué de $25 \%$ para el grupo entero de las mastectomizadas; tuvieron ganglios positivos 19 pacientes o sea un $79 \%$ y en ellas la supervivencia de 5 años fue de $21 \%$. En el segundo trimestre 15 fueron mastectomizadas y la supervivencia de 5 años fue de $33 \% ; 11$ pacientes o sea un $73 \%$ tenían metástasis ganglionares y la supervivencia de 5 años fue de $18 \%$. En el tercer trimestre 7 pacientes fueron mastectomizadas con un promedio de curaciones de $75 \%$ con ganglios axilares negativos $y$ $33 \%$ con metástasis, 73 pacientes eran puérperas y fueron mastectomizadas con $68 \%$ de curaciones en las que no presentaban invasión ganglionar contra $15 \%$ en las invadidas; 52 pacientes que habían sido sometidas a mastectomía radical quedaron posteriormente embarazadas y de éstas $38 \%$ con ganglios positivos so- 
brevivieron 3 años mientras que un $64 \%$ con ganglios negativos sobrevivieron 5 años. De lo cual se deduce que hay un pobre pronóstico cuando se encuentra invasión ganglionar cualquiera que sea la época del tratamiento en relación con el embarazo pero no puede sostenerse que sea éste el que influye en este pronóstico sino el estado de la enfermedad que es usualmente avanzado cuando se hace la mastectomía radical. Por esta razón Miller (18) afirma que si la única manera posible de curar casi todos los cánceres es llegando a un diagnóstico precoz; esto es especialmente verdad en lo que se refiere a $\mathrm{Ca}$. de seno y el obstetra debe examinar sus pacientes a este respecto frecuentemente e instruirlas para que informen sobre la aparición de cualquier nódulo.

En nuestra serie cuatro pacientes presentaban carcinoma de seno.

EI CASO No 53 una G. 15 P. 11 A. 3 de 38 años de edad con un embarazo de 32 semanas, feto vivo, carcinoma de seno izquierdo, una paciente en pésimas condiciones que rochaza tratamiento y prefiere "irse a morir a la casa".

EI CASO N: 54 una G. 6 P. 3 A. 2 de 32 años de edad con una gestación de 28 semanas, feto vivo que consultó por dolor en el seno derecho $y$ en la articulación coxo-femoral izquierda. Presenta un Ca. en seno derecho, y se remitió al Instituto Nacional de Cancerología.

EI CASO No 55 una G. 4 P. 3 de 29 años de edad con un embarazo de 40 semanas, feto vivo y $\mathrm{Ca}$. de seno izquierdo estado IV que ingresa en trabajo y tiene un parto espontáneo normal con feto vivo, femenino de 3.320 gs. de peso $10 / 10$ de Apgar, se suprimió la lactancia con estrógenos conjugados-andrógenos y se remitió al Instituto Nacional de Cancerología.

Y por último el CASO No 56 una G. 11 P. 10 de 37 años de edad con un embarazo de aproximadamente 12 semanas de evolución normal que consultó por una tumoración de seno izquierdo con dos meses de evolución, de la cual se toma biopsia y resulta un adenocarcinoma de glándula mamaria izquierda. Se remitió también al Instituto Nacional de Cancerología.

\section{2: Carcinoma de vulva y vagina.}

De por sí este tipo de carcinomas que no es frecuente, lo es mucho menos con embarazo, la mayoría de los reportes se refieren a pacientes tratados por vulvectomía radical para $\mathrm{Ca}$. que posteriormente quedan embarazadas, Collins y Barclay (19) reúnen 6 casos de Ca. vulvar asociados con embarazos y analizan 21 más de la literatura, Murphy, Wilson y Bickel (20) reportan un caso de Carcinoma adenoide quístico de la glándula de Bartolin izquierda, coexistente con embarazo. Llama la atención de estas series el hecho de que la edad de las pacientes está un poco más baja de la común del $\mathrm{Ca}$. y el promedio fué de 2 años. En la paciente del Ca. de la glándula de Bartolin se llevó a cabo primero una vulvectomía total y posteriormente una linfadenectomía inguinal izquierda. De la serie de Collins 16 habían sido tratadas y quedaron posteriormente embarazadas, a una le fue descubierto en el primer trimestre $y$ era un melanoma, tuvo parto vaginal, se le hizo linfadenectomía 2 años después y murió $3 \frac{1}{2}$ años más tarde. En 3 casos se hizo diagnóstico en el segundo trimestre todas tres fueron vulvectomizadas y tuvierón parto por Cesárea pero dos de ellas fueron por indicación obstétrica. Cinco casos fueron descubiertos en el tercer trimestre, una tratada por vulvectomía simple, parto por Cesárea, linfadenectomía y radioterapia posterior; otra recibió radium y roentgen sobre lesión y regiones inguinales, cesárea posterior. Otro caso era un sarcoma 
que fue tratado por escisión local para biopsia, parto espontáneo. El cuarto caso fue diagnosticado en el momento del parto y por último relatan un Carcinoma de vagina en una paciente de raza negra, reportado por Tuft en 1930, primigrávida, que tuvo el parto por Cesárea. Ninguna experiencia tenemos nosotros al respecto.

3․ Carcinoma de Cérvix y Utero. Superada ya la errónea idea que antes se tenía de que no debían practicarse durante el embarazo exámenes con espéculum o tacto vaginal sino en casos excepcionales, por el peligro de infección; ofrece precisamente éste la mejor oportunidad que tiene el Médico para llegar al diagnóstico precoz de las neoplasias malignas de cérvix valiéndose para ello de los métodos paraclínicos, citología, colposcopia etc. O biopsia, conización y más aún siendo el Cérvix uterino uno de los pocos sitios accesibles donde por estas ayudas se puede establecer el diagnóstico de una lesión típicamente precancerosa - preinvasiva el denominado Carcinoma in situ. Es necesario establecer primero que durante el embarazo se presentan modificaciones, no típicas, pero sí muy constantes del epitelio cervical y que además el contenido celular vaginal también participa de estas modificaciones que es preciso conocer para diferenciar las que dependen del embarazo mismo y las que pueden ser sospechosas de un proceso neoplásico incipiente.

Nesbitt (21) del John's Hopkins describe en primer lugar una hiperactividad de la capa basal del epitelio que divide en tres grados de acuerdo con el espesor de ella, en segundo lugar denomina hiperplasis glandular al aumento que encuentra en el número total de las glándulas endocervicales; si las células columnares basales están apiladas contra los tubos glandulares llama esto hiperplasia glandular epitelial y si aparecen glándulas inmaduras hiperplasia adenomatosa; encuentra también epidermización del epitelio glandular o metaplasia del epitelio columnar en epitelio escamoso estratifica. do. Macroscópicamente en 300 casos encontró 127 normales, 91 con erosión simple, 16 con infección crónica y huevos de Naboth, 64 con erosión, eversión y granulación y dos pólipos. Sobre todos los casos de biopsia encontró un $0,66 \%$ de $\mathrm{Ca}$. intraepitelial.

Por su parte Dubner (22) y colaboradores destacan la importancia del frotis de Papanicolaou durante el embarazo y llegan a sostener que con su uso puede demostrarse la progresión del cambio maligno de una displasia a un carcinoma invasivo; usándolo en forma rutinaria en el Brooklyn Hospital encontraron 18 frotis positivos entre 4.794 embarazos de 1959 al 62.

MeKelway (23) utiliza el frotis seriado en la siguiente forma: si este es grado I-II no lo repite sino 6 semanas después del parto, a menos que haya evidencia clínica de lesión pues se necesitaría estar loco para aceptar un reporte normal como seguro. El Grado III indica sospecha de malignidad pero puede ser debida a un proceso inflamatorio que trata exhaustivamente y repite el frotis dos semanas más tarde si es normal, lo repite cada tres meses, pero si vuelve a presentarse un Grado III practica biopsia o en caso de que el frotis sea Grado III persistente o Grado IV y en todo caso en que haya una lesión local sospechosa cualquiera que sea el resultado del frotis. El Grado $V$ es francamente positivo de malignidad. No gusta en 
cambio de la conización por considerarla con un riesgo de hemorragia. En el Instituto Materno Infantil apenas comienza a hacerse en forma rutinaria el Papanicolaou en las pacientes de prenatal pero fue grande nuestra sorpresa cuando en la primera semana de instaurado tropezamos con un $\mathrm{Ca}$. invasivo en paciente sin ninguna sintomatología.

La frecuencia global del Carcinoma de cérvix asociado con embarazo es muy difícil de obtener dado que los diferentes autores que se han ocupado del tema dan incidencias parciales según se trate de Carcinoma in situ o de Carcinoma invasivo y por esta misma razón los datos son muy variables y van desde el $0.005 \%$ hasta el $0,47 \%$ para diversos autores $y$ en diversas instituciones, las causas de esta diferencia tan amplia se pueden encontrar en primer lugar en tan escaso número de pacientes que cada uno reporta en sus series de tal manera que uno - dos casos más en relación con el total es definitivo en el porcentaje de incidencia; por otra parte algunas instituciones son puramente obstétricas y la relación se obtiene con el número de pacientes embarazadas atendidas, como en nuestro caso, otras son mixtas y otras por último están enteramente dedicadas a la atención de Tumores malignos y la relación se obtiene entonces al contrario con el número de pacientes con Ca. de cérvix atendidas.

En el Instituto Materno Infantil, la incidencia y los promedios de edad, gravidez, paridad y gestación en semanas están representadas en el Cuadro № 9.

\section{CUADRO № 9}

\section{AREVALO $Y$ LOZANO}

Carcinoma de Cérvix

Carcinoma escamocelular

Adenocarcinoma

Ca. muco epidermoide

TOTAL

Sobre 135.479 embarazos, 43 casos $=0,031 \%$

\section{Frecuencia}

41

1

-
Promedio de edad

Promedio de gravidez

Promedio de paridad

Promedio de gestación en semanas
34.65 años

7.95 gest.

6.58 partos

25.75 semanas

Embarazadas

Abortos retenidos sin datos de amenorrea

Aborto incompleto sin dato de amenorrea

$\mathrm{Sin}$ dato de amenorrea

TOTAL 
CUADRO N: 10

AREVALO $Y$ LOZANO

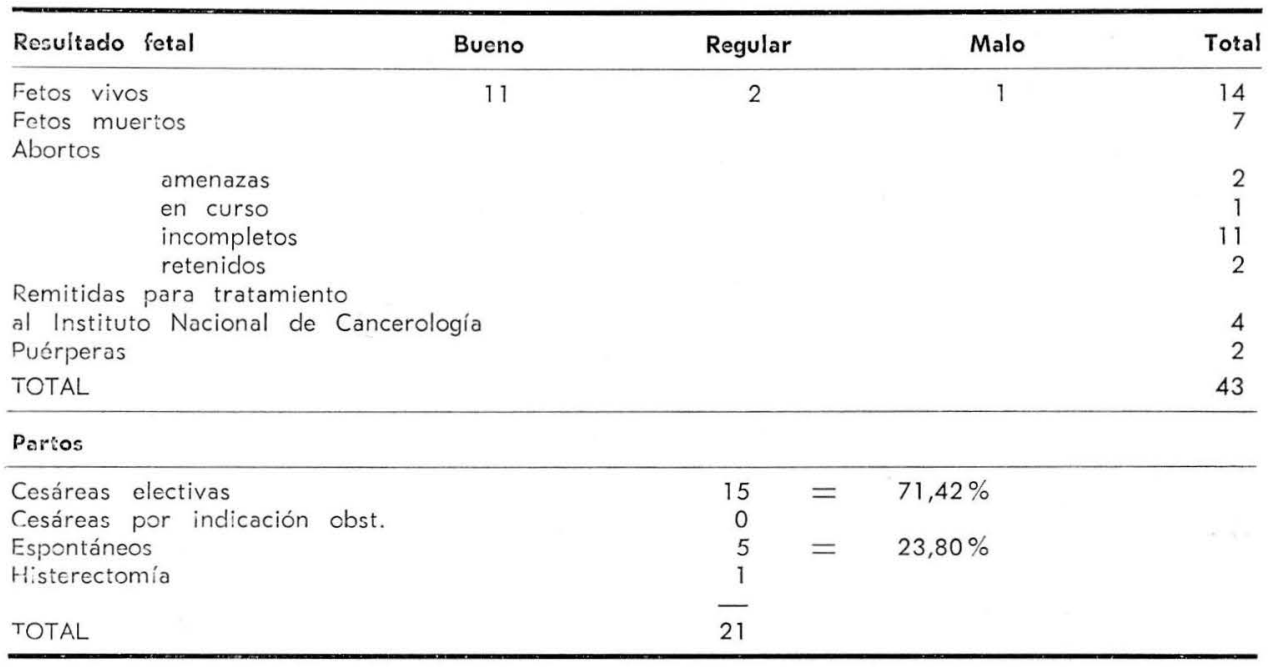

CUADRO № 11

AREVALO $Y$ LOZANO

\begin{tabular}{lrr}
\hline Sintomatología & & \\
\hline Hemorragia genital espontánea & 37 & $=$ \\
Hemorragia genital post-coito & 1 & $86,03 \%$ \\
Dolor pélvico & 16 & $=37,20 \%$ \\
Enflaquecimiento & 7 & $=16,27 \%$ \\
Flujo fétido & 4 & $=$ \\
Otros: disuria & 1 & 1 \\
$\quad$ Polaquiuria & 1 & 1 \\
$\quad$ dolor a la defecación & 1 & \\
dolor propagado a miembro inferior & 1 \\
Asintomático, hallazgo prenatal por citología y biopsia & & \\
\hline
\end{tabular}

CUADRO N: 12

AREVALO Y LOZANO

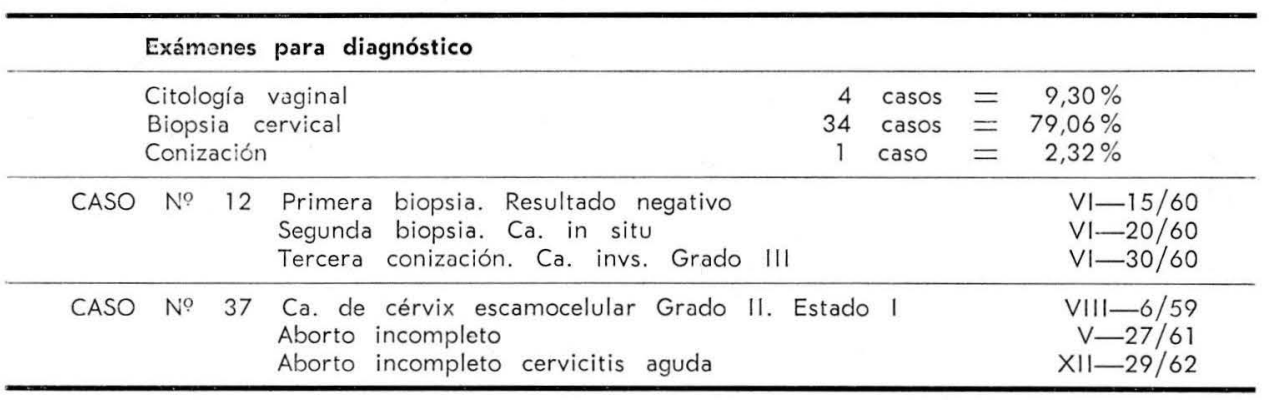


La paciente más joven de las nuestras tenía 22 años y la más vieja 45 . En relación con la gestación 2 eran puérperas y 16 tenían gestaciones de 38 semanas o más, es decir a término; 16 consultaron por alguna forma de aborto coexistente en todas ellas con un $\mathrm{Ca}$. invasivo. Los resultados fetales y partos podemos verlos en el Cuadro N: 10 y los totales de partos $y$ abortos.

La sintomatología más frecuente por la cual consultaron las pacientes queda resumida en el Cuadro No 11 y en el 12 los exámenes complementarios para Dianóstico con dos casos especiales que transcribo, por parecerme de la mayor importancia cada uno de ellos en su género, ya que en el primero se insistió en el estudio de la paciente hasta lograr comprobar la existencia de un Ca. invasivo $y$ en el segundo probablemente se trató de una confusión en la placa de la biopsia con alguna otra paciente para quien el perjuicio por ello ocasionado pueden ustedes imaginarlo.

Por lo bajo el número de casos que recibieron tratamiento de nosotros para el Carcinoma, no la conducta obstétrica, no hice una diferenciación combinada entre el estado Clínico del Ca. y la edad de la gestación en trimestres de embarazo o en primera y segunda mitad como usan otros autores para decidir la conducta terapéutica, pero en una ampliación posterior que me propongo hacer sobre los casos tratados por Cesáreas y/o Histerectomía radical lo haré detalladamente.

Vamos a detenernos ahora en algo muy importante que hace bastante diferencia y es el estado Clínico del Carcinoma y su relación con el embarazo y viceversa. Carcinoma in situ. Es numerosa la literatura al res- pecto y las discusiones que sobre el tema se han originado por los cambios histológicos del cuello durante el embarazo que hacen difícil sostener si estos cambios son atipias del embarazo o verdaderas transformaciones premalignas del epitelio cervical. Greene, Peckhan y otros (24) del Dpto. de Obst. y Gin. de la Northwestern Medical School están de acuerdo en que los cambios tan amplios del epitelio escamoso estratificado y glandular del cérvix pueden prestarse a confusión con el carcinoma preinvasivo y más cuando estos cambios no son "Específicos" del embarazo, pero en 14 pacientes a quienes se les hizo por biopsia el Diag. de Ca. in situ y que fueron seguidas durante todo el embarazo y el puerperio, las lesiones persistieron en 12 de ellas y fueron demostradas por las biopsias y las piezas quirúrgicas, luego no eran lesiones debidas al embarazo.

Los mismos Greene y Peckhan (25) en 1958 presentan una nueva serie de 19 pacientes que sumados a las 18 de reportes anteriores hacen 37 , encuentran regresión de las lesiones en 5 casos y persistencia en las demás, para un total de 29, el $78,4 \%$ de lesiones que persistieron en el post-parto, comparadas con un $78,5 \%$ de lesiones persistentes en una serie de pacientes no embarazadas lo cual los lleva a concluir que "el embarazo no tiene el mágico poder de mimetizar el Ca. preinvasivo y el diagnóstico de estas lesiones es válido durante él". De la misma opinión son Osband y Nicholson Jones (26) de la Univ. de Alabama, los cuales insisten también en el valor del test de Papanicolaou, practican la conización para confirmar el estado $O$, permitiendo en este caso el parto vaginal y en el post-partum terapéutica definitiva por conización o 
histerectomía. Dean, Isbell y Woodard (27) hacen énfasis también en el valor del Papanicolaou encontrando que una de cada 142 pacientes de una consulta prenatal de 8939, tenían células exfoliativas anormales en vagina y de éstas, dos tercios comprobaron tener $\mathrm{Ca}$. in situ $\mathrm{O}$ invasivo en cérvix; en la citología positiva persistente practicaron biopsia y en caso de comprobar el estado preinvasivo fueron tratadas conservadoramente permitiendo el parto vaginal a menos que existieran indicación obstétrica, seguida de nuevas biopsias alrededor de 4 meses después del parto e Histerectomía en caso de persistencia de la enfermedad.

En nuestra literatura no encontré reportes de Carcinoma preinvasivo y de los casos reportados en esta serie del Instituto Materno Infantil el único que tuvo este Diagnóstico previamente fue confirmado posteriormente como Estado I en la pieza quirúrgica.

Carcinoma Invasivo del Cérvix. En el Chicago Lying Hospital en 1956, Hayden (28) sobre 81.806 embarazos encontró 12 casos de $\mathrm{Ca}$. de cérvix, incidencia del $0,014 \%$; la relación del embarazo con el Ca. fue de 12 para 485 casos de neoplasia cervical el $2,47 \%$. Los síntomas fueron semejantes a los de cualquier malignidad del cérvix más los propios del embarazo, destacándose la hemorragia vaginal en el $91,6 \%$ de los casos, $25 \%$ eran Estados 1, 58,3\% eran Estados II y $16,6 \%$ eran Estados III con una supervivencia materna de 5 años del $50 \%$ en los estados I y 11 y $0 \%$ en el III. Practicaron biopsias de rutina en los casos necesarios durante la investigación prenatal, el más importante criterio pronóstico fue el estado clínico, la mortalidad fetal no se encuentra sino muy ligeramente aumentada por el Carcinoma.
No pueden demostrar influencia desfavorable del embarazo mismo sobre las cifras de supervivencia y el método de tratamiento escogido fué la Cesárea con Histerolinfadenectomía en los Estados I y II pero si había invasión extensa del cuello hicieron radiumterapia previa seguida de Cesárea y, o Cirugía radical. En un solo caso en una paciente con una gestación de 12 a 14 semanas hicieron Histerectomía sub-total seguida de radiumterapia. En el magnífico artículo de Betson (6) tantas veces citado presenta 30 casos de Carcinoma de cérvix y embarazo de los cuales 19 fueron no invasivos y 11 invasivos en 8 de los cuales se practicó Cesárea con feto vivo, un aborto espontáneo en las 12 semanas de gestación, una Histerectomía radical después de radiumterapia en las 22 semanas y otra histerectomía con linfadenectomía pélvica. Las pautas de tratamiento dependen del trimestre de gestación y el estado Clínico así: en el primer trimestre Estados I y II, Histerectomía radical, Estados III y más, radioterapia o roentgenterapia profunda, esperar el aborto y luego radiumterapia 2 a 4 semanas después. En el segundo trimestre aplican radium y evacúan posteriormente el útero por histerotomía abdominal, luego hacen radiumterapia una semana más tarde. En el tercer trimestre aplican radium entre la 29 y 34 semanas de gestación, seguida de cesárea clásica tan pronto consideran viable el feto, posteriormente roentgenterapia o radium en cuanto cura la cicatriz abdominal; en el puerperio tratan con Rayos $\mathrm{X}$, Radium o $\mathrm{Ci}$ rugía radical.

Waldrop y Palmer (29) del Roswell Park Memorial Institute, Buffalo, revisan 6.000 casos de carcinoma cervical entre 1919 y 1955 sus conclusiones en cuanto a incidencia, sín- 
tomas y diagnóstico son semejantes a las de los autores anteriormente citados, afirman que solo excepcionalmente el embarazo influye en el crecimiento tumoral, que los promedios de curación son idénticos en la primera y segunda mitad del embarazo aunque frecuentemente en su medio el diagnóstico se hace precozmente cn el embarazo y los estados clínicos son mejores que en las pacientes no embarazadas, no hacen cirugía sino roentgen o radiumterapia y comparan los resultados con los de aquella, la supervivencia es tan buena o mejor que en las pacientes no embarazadas y por último no estiman que haya ninguna contraindicación para el parto vaginal.

Muy al contrario Barber y Brunschwig (30) del Memorial Hospital for Cancer y James Ewing Hosp. dan un porcentaje global de $56 \%$ de curaciones para todas las edades y estados clínicos globales tratados únicamente con Cirugía pero con mejor pronóstico para las intervenidas durante el cmbarazo que en el puerperio; Kinch (31) en una comparación de 75 pacientes embarazadas con Ca. de cérvix y 402 no embarazadas del mismo grupo de edades y estadios clínicos dá un porcentaje de supervivencia de 5 años muy semejante de 44 a $45 \%$ respectivamente; sinembargo es enfático en declarar que mientras más precoz el estado clínico, más precoz el embarazo y más precoz el tratamiento mejores resultados se consiguen, sugiere que por algunos casos desafortunadamente de Estados I el parto vaginal con el consiguiente traumatismo cervical que ocasiona produce una rápida diseminación de la enfermedad, los peores resultados cn los casos diagnosticados y tratados inmediatamente después del trabajo $y$ en el puerperio.

Los casos avanzados en el emba- razo precoz los trata con Rayos $X$, Cobalto, espera el aborto y aplica Radium en dosis cancericida. Los casos recientes en embarazo en los primeros meses se les puede aplicar el mismo tratamiento anterior o la cirugía radical con linfadenectomía por cirujanos competentes. Los casos tardíos diagnosticados durante el trabajo de parto Cesárea seguida de radioterapia adecuada comenzando por Rayos $X$ profundos o Cobaltoterapia, en los Estados Tempranos durante el trabajo puede darse el mismo tratamiento o terminar con un manejo quirúrgico radical adecuado después de la Cesárea. El diagnóstico en el segundo trimestre impone una gravo responsabilidad puesto que la concurrencia del embarazo nos debe llevar a obtener un feto capaz de sobrevivir.

En el trabajo de Jordan y Darnalt (1) presentado en 1955 estudian 10 casos de $\mathrm{Ca}$. de cérvix y embarazo, revisan la incidencia de varios autores y respecto al tratamiento siguen las pautas que expusimos en la cita anterior, vigilancia y observación en los Estados 0, cirugía radical en los Estados I y II diagnosticados precozmente en el embarazo sin que cuente la vida fetal sino la materna o esperar al máximo de viabilidad en la segunda mitad de la gestación para proceder a Cesárea e Histerolinfadenectomía. En estados avanzados tratamiento actinoterápico en los primeros meses de embarazo, y Cesárea, y lo mismo en la segunda mitad. López Escobar, Buendía, Gaitán Yanguas y Rosas (32) encontraron 11 casos de $\mathrm{Ca}$. de cérvix y embarazo sobre 600 carcinomas con una frecuencia de 1,83\%, ningún Estado 0, dos Estados I, III y IV y cinco Estados II. Por ser un trabajo puramente estadístico, no dan ningún dato sobre tratamiento y pronóstico. Tirado 
Vélez (3) en 1959 nos informa sobre su intervención directa en 3 casos de Ca. de cérvix y embarazo con sus planteamientos y conducta nos encontramos completamente de acuerdo. Por último James A. O'Leary (33) del Sloane Hospital for Women en el Columbia Presbyterian Medical Center, New York City, presenta 6 casos de Carcinoma de cuello y embarazo, todos Estados I y II (un caso) todos entre la 25 y 36 semanas de gestación, tratados 5 por Cesárea seguida de Histerectomía radical con linfadenectomía Pélvica Bilateral y uno con Histerotomía abdominal y cirugía radical, una fístula urétero-vaginal y otra vésico-vaginal como complicaciones, entre $5 \frac{1}{2}$ y 7 horas de tiempo quirúrgico, 4 fetos vivos, dos muertes neonatales.

En nuestra serie el Diagnóstico del Estado Clínico del $\mathrm{Ca}$. al ingreso y a la salida de la paciente está resumido en el Cuadro No 13. El caso diagnosticado como $\mathrm{Ca}$. no invasivo al ingreso era Estado I a la salida y 2 de los Estados I fueron con meiástasis ganglionares, Estados III. Como exámenes complementarios para el tratamiento quirúrgico se practicaron Radiografía de Tórax en 13 casos, de pelvis en 2 , de contenido uterino en 1. Cistoscopia en 6 casos, rectosigmoidoscopia en 10 y estudio cardiovascular preoperatorio en $1 \mathrm{ca}$ so.

Los tratamientos quirúrgicos que recibieron las pacientes están resumidos en el Cuadro No 14 al cual quiero aclararle que la Cesárea y la Histerectomía radical practicada en el puerperio fué una conducta obligada en una paciente $R h$ negativa que no se tuvo sangre de ese tipo para operar en el momento del parto por lo cual fué necesario hacer primero la Cesárea y luego la Histerolinfadenectomía cuando se consiguió la sangre; la Histerectomía radical en el puerperio fue una paciente que ingresó en período expulsivo y tuvo un parto espontánco, se trató con cirugía radical posteriormente. La Histerectomía total del caso $N$ ㅇ 16 era una paciente con feto muerto y una gran infección amniótica, se sacó el útero con todo y feto y se remitió al Instituto de Cancerología para el tratamiento de un Estado I. La histerectomía total ampliada fue el Carcinoma in situ que en la pieza resultó un Invasivo, se le practicó Cesárea e Histerectomía y se envió luego a Radioterapia. El resultado de las piezas Quirúrgicas está resumido en el Cuadro № 15.

La conducta Obstétrica está en un todo de acuerdo con la opinión de

\section{CUADRO N: 13}

AREVALO Y LOZANO

\begin{tabular}{ccc}
\hline Diagnóstico del cstado clínico & Ingreso & Salida \\
\hline Carcinoma in situ & 1 & 0 \\
Ca. estadio I & 15 & 14 \\
Ca. estado II & 7 & 7 \\
Ca. estado III & 16 & 18 \\
Ca. estado IV & 3 & 3 \\
Desconocido & 1 & 1 \\
TOTALES & 43 & 43 \\
\hline
\end{tabular}

El carcinoma in situ al ingreso fué estado I a la salida.

Dos casos de estado I al ingreso fueron estado III a la salida. 


\section{CUADRO NN 14}

\section{AREVALO $Y$ LOZANO}

Tratamientos y estados clínicos

Cesárea con Histerectomía radical. Estado I

Cesárea con Histerectomía radical. Estado II

Cesárea con Histerectomía radical en puerperio. Estado I

Parto espontáneo con Histerectomía radical en el puerperio. Estado I

Histerectomía radical con embarazo (12 semanas) Estado I

Raspado uterino. Histerectomía radical. Estado I

Histerectomía total ampliada. Estado 0

Histerectomía total simple. Estado I

TOTAL

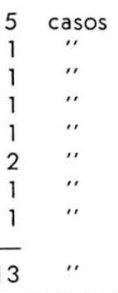

\section{CUADRO № 15}

\section{AREVALO Y LOZANO}

\begin{tabular}{l}
\hline Resultado histopatológico de piezas quirúrgicas \\
\hline Carcinoma in situ \\
Carcinoma estado I \\
Carcinoma estado II \\
Carcinoma estado III \\
Caso No 8. Histerectomía radical en el IX día \\
del puerperio después del parto espontáneo \\
TOTAL
\end{tabular}

la mayoría de los autores que hemos citado; los casos I y II que se diagnostiquen en la primera mitad de la gestación deben ser tratados como si no existiera el embarazo ya que esperarse a la viabilidad fetal sería sumamente arriesgado para la madre, así practicamos una Histerectomía radical con un embarazo de 12 semanas; los estados clínicos más avanzados fueron enviados todos al Instituto de Cancerología para Actinoterapia fuera embarazadas o con Abortos.

En la segunda mitad del embarazo dejamos llegar el feto a las máximas condiciones de viabilidad y se intervino por Cesárea, seguida de Histerolinfadenectomía en los Estados I y II (uno), en muchos de los Estados más avanzados se hizo Cesá- rea electiva precoz y se remitió la paciente después para Actinoterapia; sinembargo, es de lamentar que por falta de suficiente criterio on algunos de los residentes, varios de estos casos con hospitalización muy anterior al parto se les permitió éste en forma espontánea con el consiguiente peligro de diseminación. Los dos que fueron operados en el puerperio quedaron ya explicados.

En cuanto a los resultados presentamos los que hemos podido reunir (Cuadro № 16) sabemos bien lo difícil que es en nuestro medio y particularmente en las pacientes del tipo hospitalario del I. M. I., conseguir que vengan a controles, por la premura del tiempo al momento de concluir este trabajo no logramos hacernos a los controles de las pacientes 
CUADRO NN 16

AREVALO $Y$ LOZANO

\begin{tabular}{|c|c|c|c|c|c|}
\hline Controles & Bien & Repro. & Pos. Muer. & Perdidas & Total \\
\hline De 5 años & 2 & & & & 2 \\
\hline de 4 años & $\overline{1}$ & & & & 1 \\
\hline de 2 a 3 años & 2 & & & & 2 \\
\hline en el primer año & 2 & 1 & 1 & 4 & 8 \\
\hline TOTALES & 7 & 1 & 1 & 4 & 13 \\
\hline
\end{tabular}

\section{Complicaciones}

Fístula urétero-vaginal

Fístula urétero y vésico-vaginal

Eventración post-operatoria

Absceso para-rectal derecho

TOTAL

que por uno $u$ otro motivo se remitieron al Instituto de Cancerología pero cuando ampliemos este estudio en lo que a Ca. de cérvix y embarazo se refiere, tarea que nos hemos propuesto para muy pronto, quizás podamos allegar los datos al respecto.

\section{Complicaciones. Cuadro № 16.}

4. Carcinoma de Ovario. Dijimos antes que en nuestra manera de ver la coexistencia de Ca. de ovario y embarazo es sumamente rara pues lo primero que sucede en este tipo de neoplasia es la destrucción de la gónada funcionalmente y por consiguiente la esterilización cuando no la incidencia sobre el estado general de la paciente con el mismo resultado; sinembargo, Barber y Brunschwig (30) refieren 10 casos, nueve diagnosticados durante el embarazo y uno al 4 : mes del puerperio, tres tuvieron supervivencia de 5 años y uno de 2, seis murieron. Munnell (34) menciona los Cistadenocarcinomas pseudomucosos y sero-papilares de baja malignidad como el Carcinoma primitivo de ovario limitado a éstos exclusivamente, como hallazgos de laparotomía en el curso de un embarazo, seguidos de ooforectomía uni
- bilateral y con una supervivencia notable de hasta el $60 \%$ en 5 años; permitiendo la continuación del embarazo y su terminación feliz.

Descrito por Donald Brock (35) de Detroit, Mich., encontramos un caso de Tumor de Cels de la granulosa asociado al embarazo, en una paciente de 23 años de edad, a pesar de lo relacionado que se encuentra dicho tumor con la esterilidad por la inhibición hormonal de la ovulación que produce. Fue intervenida por síntomas de ruptura del tumor con un embarazo de 7 meses cuyo parto se produce después de la operación con muerte fetal. Nosotros no tenemos ninguna experiencia al respecto.

5: Sarcomas genitales. No mencionaremos referencias sobre sarcomas de vulva y vagina pues no tienen utilidad en este trabajo. Los tumores conjuntivos malignos de útero y cérvix de por sí muy raros entre todas las neoplasias de estos órganos lo son excepcionales coexistiendo con un estado grávido-puerperal. Corscaden (36) estudia 57 casos de sarcomas uterinos en el Sloane Hospital, no hace ninguna mención de coexis- 
tencia con embarazo, en 5 observaciones de sarcomas uterinos Normando Arenas (37) hace consideraciones sobre la frecuencia, edad, paridad etc., tres pacientes eran menopáusicas, ninguna relación con embarazo. En la revisión de López Escobar y McLean (38) de las Historias del Instituto de Cancerología de 1935 a 1959 encontraron 20 casos que analizan exhaustivamente, entre ellos un Rabdomiosarcoma en una paciente de 45 años de edad. Ninguna relación con embarazo, ni tampoco en el trabajo presentado por el Dr. Pelayo Correa (39) al Simposio sobre el Cáncer del Utero celebrado en Méjico; la única referencia positiva al respecto la encontramos en un artículo de Díaz Bazan y Roberto Masferrer (40) quienes reportan un caso de Sarcoma cervical asociado con embarazo y hacen una revisión de la literatura. Nosotros presentamos un interesante caso el № 10, una paciente de 18 años de edad G1 PO el que consultó por hemorragia genital de más o menos 15 días de duración y que un año antes había sido sometida a una tiroidectomía subtotal por un Ca. mixto folicularpapilar de tiroides. Se practicó Raspado Uterino y Biopsia de una tumoración cervical. Encontraron restos ovulares de un aborto incompleto y reportaron como resultado de la biopsia un Leiomiosarcoma de Cérvix. Se hizo luego Histerectomía total con salpingo-ooforectomía bilateral y en la pieza quirúrgica reportaron Rabdomiosarcoma de cérvix. Se remitió posteriormente al Instituto Nacional de Cancerología pero la paciente murió a los pocos meses con metástasis. Mencionamos para terminar los cuatro casos de Coriocarcinoma y los 2 de Corioadenoma Destruens que encontramos en nuestra casuística. Hasta aquí el presente trabajo con toda la literatura que hemos podido allegar al tema de malignidad y embarazo o como nosotros lo reformamos por las razones aducidas al principio del Embarazo y Malignidad y la pequeña serie con que contribuímos a aumentar la casuística nacional.

\section{COMENTARIOS}

Nuestro propósito al ejecutar este trabajo no fue de ninguna manera aportar hechos nuevos, sino únicamente efectuar una revisión bibliográfica de más o menos 10 años, que peca naturalmente por incompleta pues en lo internacional casi toda se reduce a la literatura norteamericana al respecto ya que nos fue imposible agregar referencias latinas a pesar de que las solicitamos al servicio de Información Bibliográfica de Ciudad de México pero aun no nos ha Ilegado; y agregar una nueva se-

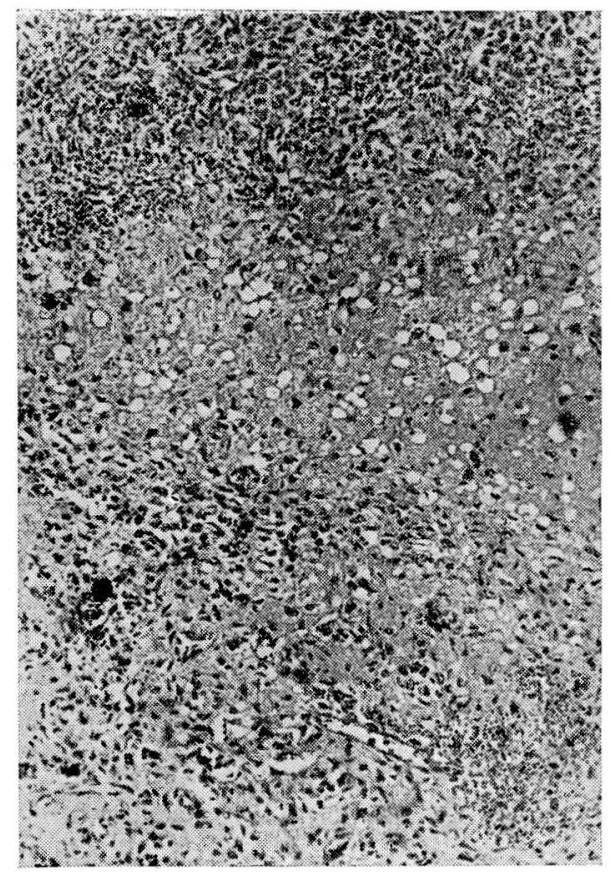




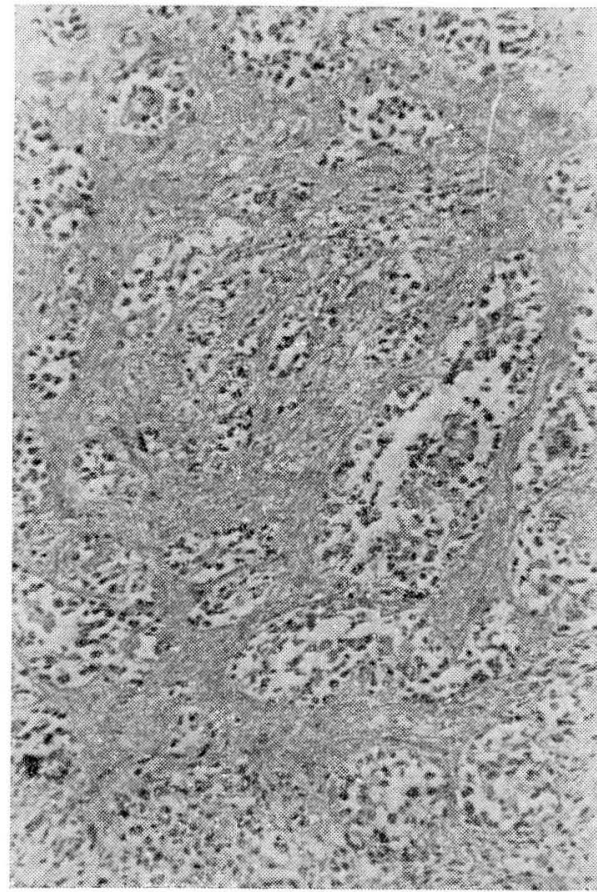

rie, eso sí, creemos hasta ahora la más alta en lo que se refiere a coexistencia de malignidad de cualquier tipo con embarazo, de las conocidas, producto de las estadísticas de un centro obstétrico puro, nacional, seguramente el de más alto número de ingresos de pacientes por año, hecho que se destaca al estudiar los cuadros del material recolectado. Así mismo y por tratarse de un tema de tan vasta envergadura y tan sumamente amplio quisimos enfocar el problema desde un punto de vista de un denominador común, el embarazo, en relación con la malignidad coexistente, en diversos órganos pero afectándolo a éste de una manera directa como cuando se asentaba en genitales o glándula mamaria, 0 al contrario afectando el embarazo cuando su localización era extragenital o mamaria o por último con una evidente relación con el estado grávido-puerperal en el coriocarcinoma. Como no es frecuente la asociación, 60 casos no son muchos pero de ninguna manera son pocos en un trabajo de este tipo, las relaciones de frecuencia, global y parcial, edad, paridad etc. son comparables con las de los autores extranjeros citados y con excepción del Carcinoma de cuello, el número de casos tan bajito no permite hacer ningún comentario, sino simplemente la citación dentro del trabajo. Respecto a éste merece un análisis más detallado. No es ni en mucho, un número de pacientes que constituyen una casuística suficiente para sacar conclusiones pero sí constituyen un aporte notable, especialmente tratándose de una entidad asistencial típicamente obstétrica; las relaciones y promedios son comparables con los de la literatura consultada, debemos destacar la presencia de hemorragia genital espontánea como síntoma predominante y el dolor pélvico aunque este último nos parece de difícil valoración, sobre todo atribuído exclusivamente como signo de malignidad pues bien puede deberse a los que acompañan frecuentemente el embarazo por atirantamiento de los ligamentos redondos; de la misma manera el enflaquecimiento tampoco puede valorarse como exclusivamente debido a la evolución de la Neoplasia, mientras que el flujo fétido sí y éste no lo encontramos sino en un $9,30 \%$ de los casos. De los exámenes practicados para diagnóstico es criticable el escaso número de citologías vaginales pero desgraciadamente en aquella época no contábamos con citólogo preparado que pudiera practicarlas, en la actualidad se está haciendo de rutina en la consulta Prenatal.

De los estados clínicos aquellos que hicimos figurar inicialmente co- 


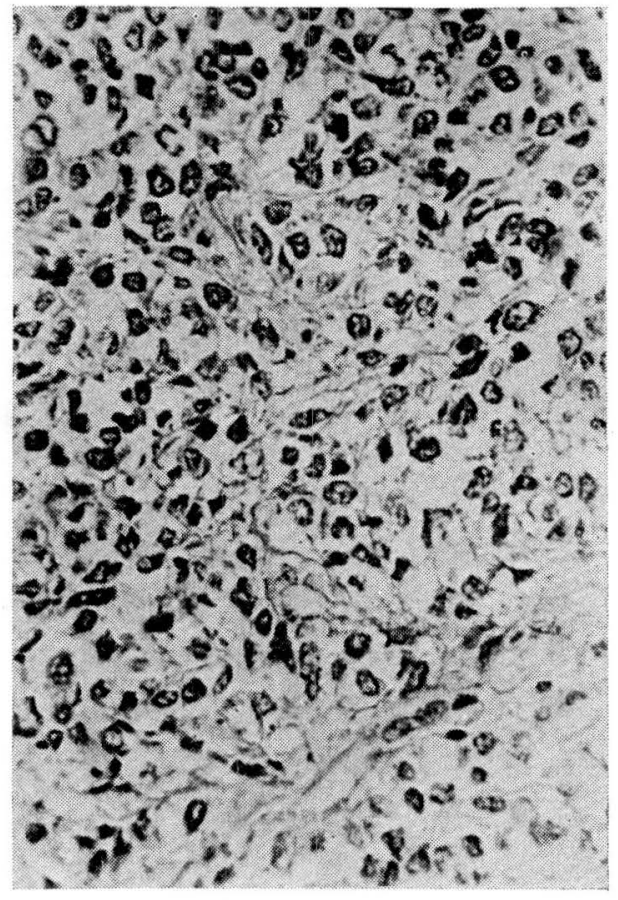

mo Estados I y cuyos ganglios linfáticos fueron metastásicos continúan desde el punto de vista de la clasificación clínica siendo Estados I, pero para remitir a tratamiento complementario en el Instituto de Cancerología eran Estados III; el Estado 0 que a la postre resultó invasivo por ser una pura clasificación histológica sí lo clasificamos de salida como Estado I. La mayoría de las pacientes fueron casos cuya clasificación clínica no permitía el tratamiento por nosotros en el Materno-Infantil y por ello de los 43 presentados 30 fueron remitidos al Instituto de Cancerología para irradiación o tratamiento paliativo, de las restantes 13 , una sola, la de la Histerectomía total simple en un Estado I fue un tratamiento parcial complementando posteriormente con irradiaciones, en los demás casos se aspiró lograr un tratamiento radical y muchos de ellos fueron personalmente intervenidos por el primero de los autores de este trabajo, sin embargo, su análisis, conducta obstétrico-quirúrgica y hasta una película en colores serán objeto de una comunicación más extensa e importante que presentaré posteriormente en la cual aspiramos también a tener controles más completos dentro de la medida tan limitada que da nuestra clientela hospitalaria; solo quiero dejar aquí la constancia de que en nuestro concepto la gestación no hace más complicado el acto quirúrgico y antes bien me atrevería a afirmar que de cierta manera lo facilita.

En lo que se refiere a las complicaciones el porcentaje, alrededor del $15 \%$, de complicaciones urológicas es sensiblemente igual al que han tenido nuestros colegas del servicio de Ginecología.

Habiendo terminado ya este trabajo se practicó una revisión de las placas del Rabdomiosarcoma Uterino que mencionamos y nuevos cortes (placas) que demostraron no solamente áreas características de esta neoplasia sino también de diferentes tejidos, Leio y algunas que recuerdan en algo el tejido neural, inclusive áreas indiferenciadas que no permiten clasificación por lo cual se cambió el parecer del patólogo hacia un Tumor mixto sarcomatoso de origen Mülleriano.

Como anunciamos al principio los casos de coriocarcinoma solo los presentamos sin hacer ningún comentario sobre ellos.

\section{SUMARIO}

1. Sobre 151.144 ingresos en $7 \frac{1}{2}$ años al Instituto Materno Infantil de los cuales 135.479 estaban embara- 
zadas encontramos 60 casos con malignidad.

2. Enfocamos el problema desde un triple punto de vista en relación con la influencia de la malignidad sobre el embarazo, de éste sobre aquella y con el Coriocarcinoma, patrimonio exclusivo del estado grávido-puerperal.

3. Primero en relación con la malignidad en general coexistiendo con el embarazo y luego desde cada uno de los puntos de vista desde los cuales enfocamos el problema, revisamos la literatura nacional y foránea al respecto.

4. Pasamos revista a las neoplasias malignas con asiento en los diversos órganos genitales, extra-genitales y mamarios coexistentes con el embarazo, señalamos sus frecuencias, promedios etc. en nuestra serie y en los artículos consultados, su sintomatología más frecuente y su conducta obstétrica y terapéutica.

5: Presentamos los resultados fetales, los embarazos que terminaron en abortos y las puérperas.

6: Hacemos un hincapié mayor en todos aquellos datos mencionados anteriormente en pacientes que tuvieron Diagnóstico de Ca. de cuello y embarazo con anotación de los principales síntomas, exámenes complementarios, resultados fetales, tratamiento, estudios Histopatológicos y controles.

7. Por último, por ser tan escaso el número de casos, aunque no pocos para el tipo de estudio emprendido, no expresamos conclusiones y nos limitamos a algunos comentarios al respecto.

\section{BIBLIOGRAFIA}

1 JORDAN S., DARNALT E.: Memorias de la $2^{\text {a }}$ Convención Col. de Obst. y Ginec. Nov.
29 Dbre. 3 1955. Edición especial de la Revista Col. de Obs. y Ginec.

2 GAITAN YANGUAS M.: Relaciones mutuas entre el embarazo y la Enfermedad de Hodgkin. Rvta. Col. de Obs. y Ginec. Vol. IX. № 3: 293. Mayo-Junio 1958.

3 TIRADO-VELEZ L.: Carcinoma de Cérvix y embarazo. Boletín científico de la Clínica de Maternidad "Luz Castro de Gutiérrez". Año II N: 5 Novbre. 1959.

4 HARBISON S. P.: Gastrointestinal and $\mathrm{Bi}$ liary tract malignancy associated with pregnancy. $\mathrm{Cl}$. Obst. and Gyn. Vol. $6 \mathrm{~N}$. 4: 1002 Dbre. 1963.

5 HESSELTINE H., LOTH M. F.: Malignant diseases associated with Pregnancy. Year Book of Obs. and Gyn. 102 1957-1958. Greenhill.

6 BETSON J. R., Jr. GOLDEN M. L. : Cancer and Pregnancy. Am. J. of Obs. y Gynec. 81: 7181961.

7 VELEZ A., JUBIZ A.: Carcinoma de la mama en el embarazo y la lactancia. Rev. Col. de Obst. y Ginec. XIV. № 5 Sept. Oct. 1963.

8 GREENSPAN, GERSON, LESNICK: Pregnancy and Cancer Medical, Surgical and Gynecological complications of Pregnancy. Staff of the Mount Sinai Hospital. New York. Williams and Wilkins. Baltimore 1960. Pág. 559.

9 FELTER T. R., KOPPEL M. M.: Urologic Malignancy Associated with Pregnancy. Clin. Obst. and Gynec. 6: 1.011 № 4. 1963.

10 WADDINGTON H. K.: Renal Cell. carcinoma complicated by Pregnancy. A. J. Obst. y Gynec. 72: 4411956.

11 BARRY R. M., DIAMOND H. D., CONVERS L. F.: Influence of Pregnancy on course of Hodgkins disease. Year Book of Obst. Y Gynec. 128 1963-1964. Greenhill.

12 HENNESSY J. P., ROTTINO A.: Hodgkins disease in Pregnancy. Am. J. Obst. \& Gynec. $87: 8531963$.

13 PECK S. M., KANTOR I.: Dermatologic Complications of Pregnancy. Staff of the Mount Sinai Hospital. New York City. Williams and Wilkins. Baltimore 1960. Pág. 470.

14 BARON C.: Melanoma and Pregnancy. Am. J. Obst. \& Gynec. 81: 10 42. 1961. 
15 MCCALL: Malignancy associated with Pregnancy. Cli. Obst. y Gynec. 6: 925. 1963.

16 TAYLOR T. Ca. of the Breast in the Pregnant and the Nursing patient. Am. J. Obst. \& Gynec. 69: 1277 al 1284. 1955.

17 HOLLEB A. J., FARROW J. H.: The relation of Carcinoma of the Breast and pregnancy in 283 patients. Surg. Ginec. and Obst. 115: 65.1962.

18 MILLER H. K.: Cancer of the Breast during Pregnancy and Lactation. Am. J. Obst. \& Gynec. 83: 6081962.

19 COLLINS C. G., BARCLAY, D. L.: Cancer of the vulva and Cancer of the vagina in Pregnancy. Cl. Obst. \& Gynec. 6: 927. 1963.

20 MURPHY J. WILSON J. M., BICKEL D. A. : Adenoid Cistic Carcinoma of the Bartholin Gland and Pregnancy. Obst. \& Gynec. 83: 612. 1962.

21 NESBITT R. E. L. Jr., HELLMAN L. M. : The Histopatology and Citology of the Cervix in Pregnancy. Surg. Gynec. \& Obst. 94: 14. 1952.

22 DUBNER M. S., ROSATI V., DUCKMAN S.: The positive Papanicolaou smear in Pregnancy. Am. J. Obst. \& Gynec. 88: 17. 1964.

23 MCKELWAY W. P.: Ca. of the Cervix in the first trimester of Pregnancy. Cli. Obst. \& Gynec. 6: 945. 1963.

24 GREENE R. R., PECKHAM B. M., CHUNG J. T., et. al. Preinvasive Ca. of the cervix during Pregnancy. Surg. Gynec. \& Obst. 96: 71. 1953.

25 GREENE R. R., PECKHAM B.: Preinvasive Ca. of the cervix and Pregnancy. Am. J. Obst. \& Gynec. 75: 551. 1958.

26 OSBAND R. NICHOLSON JONES. W. Ca. in situ in Pregnancy. Am. J. Obst. \& Gynec, 83: 605. 1962.

27 DEAN R. E., ISBELL N. P., WOODARD D. E.: Cervical Ca. in Pregnancy. Obst. \& Gynec. 20: 635. 1962.
28 HEYDEN G. E.: Ca, of the Cervix associated with Pregnancy. Am. J. Obst. \& Gynec. 71: 780. 1956.

29 WALDROP G. M. PALMER J. P. Ca. of the Cervix Associated with Pregnancy. Am. J. Obst. \& Gynec. 86: 202. 1963.

30 BARBER H. R. K. BRUNSCHWIG A.: Gynecologic Cancer complicating. Pregnancy. Am. J. Obst. \& Gynec. 85: 156. 1963.

$31 \mathrm{KINCH}$ R. Factors affecting the prognosis of Cancer of the cervix in Pregnancy. Am. J. Obst. \& Gynec. 82. 45. 1961.

32 LOPEZ-ESCOBAR G., BUENDIA FERRO A., GAITAN-YANGUAS M., ROSAS A.: Cáncer del útero en Colombia. Boletín del Comité Nal. de lucha contra el cáncer. 10: 28 Marzo 1964.

33 O'LEARY J. A.: Radical Pelvic Surgery in Pregnancy: the radical cesarea Histerectomy. Bull. of the Sloane Hospital for Women. Vol. XI. Spring. 1965.

34 MANNELL E. W. Primary ovarian cancer associated with Pregnancy. Cli. Obst. \& Gynec. 6: 983. 1963.

35 BROCK D. R. Granulosa cell tumors of the ovary associated with Pregnancy. Am. J. Obst. \& Gynec. 83. 109. 1962.

36 CORSCADEN J. A. SINGH B. Leiomiosarcoma of the Uterus. Am. J. Obst. \& Gynec. $75: 149.1958$.

37 ARENAS N., FOIX A., DAWSON T. Sarcomas del útero. Aporte de 5 observaciones. Rvta. Argentina de Cancerología. Vol. 2 N: 3: 146. 1960.

38 LOPEZ-ESCOBAR G., MCLEAN J, : Sarcomas del útero. Rvta. Col. Obst. \& Gynec. Vol. XI. No 4: 339. 1960.

39 PELAYO CORREA. Patología de los tumores malignos uterinos. Boletín del Comité $\mathrm{Nal}$. de lucha contra el cáncer. 11: 3. 1964.

40 DIAZ BASAN., MASFERRER R.: Cervical Sarcoma associated with Pregnancy. Review of the literature and report of case. Year Book of Obst. and Gynec. 113. 1961. 1962. 